\title{
PUMP-FREE MICROFLUIDIC DEVICE FOR THE ELECTROCHEMICAL DETECTION OF $\alpha_{1^{-}}$ACID GLYCOPROTEIN
}

Tania Sierra ${ }^{1,2}$, Ilhoon Jang1,3, Eka Noviana ${ }^{1,4}$, Agustín G. Crevillen ${ }^{5}$, Alberto Escarpa $^{2,6^{*}}$, Charles S. Henry ${ }^{1 *}$

${ }^{1}$ Department of Chemistry, Colorado State University, Fort Collins, C0, 80526, USA

${ }^{2}$ Department of Analytical Chemistry, Physical Chemistry and Chemical Engineering, University of Alcala, E-28871, Alcala de Henares, Madrid, Spain.

${ }^{3}$ Institute of Nano Science and Technology, Hanyang University, Seoul, 04763, Korea

${ }^{4}$ Department of Pharmaceutical Chemistry, Faculty of Pharmacy, Universitas Gadjah Mada, Yogyakarta 55281 Indonesia

${ }^{5}$ Department of Analytical Sciences, Faculty of Sciences, Universidad Nacional de Educación a Distancia (UNED), E-28040, Madrid, Spain.

${ }^{6}$ Chemical Research Institute “Andrés M. del Río” (IQAR), University of Alcala, E-28805 Alcala de Henares, Madrid, Spain.

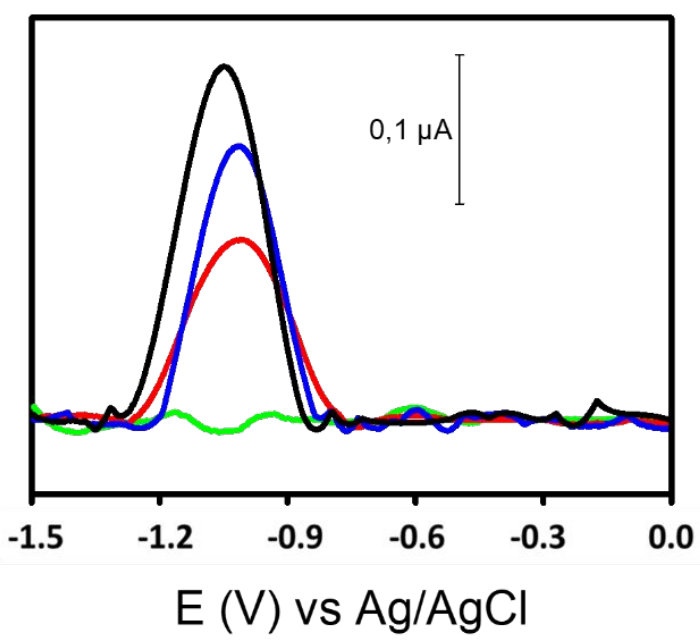

Figure S1. Voltammograms of $2000 \mathrm{mgL}^{-1} \mathrm{AGP}-\mathrm{Os}(\mathrm{VI})$ by AdTSWV using different incubation times. Green line: $0 \mathrm{~min}$; red line: $10 \mathrm{~min}$; blue line: $20 \mathrm{~min}$ and black line: 30 min. SWV parameters: start potential $-1.5 \mathrm{~V}$, end potential $+0.0 \mathrm{~V}$, step potential $5 \mathrm{mV}$, amplitude $50 \mathrm{mV}$, and frequency $2 \mathrm{~Hz}$ (electrochemical signals were baseline corrected and smoothed out). 

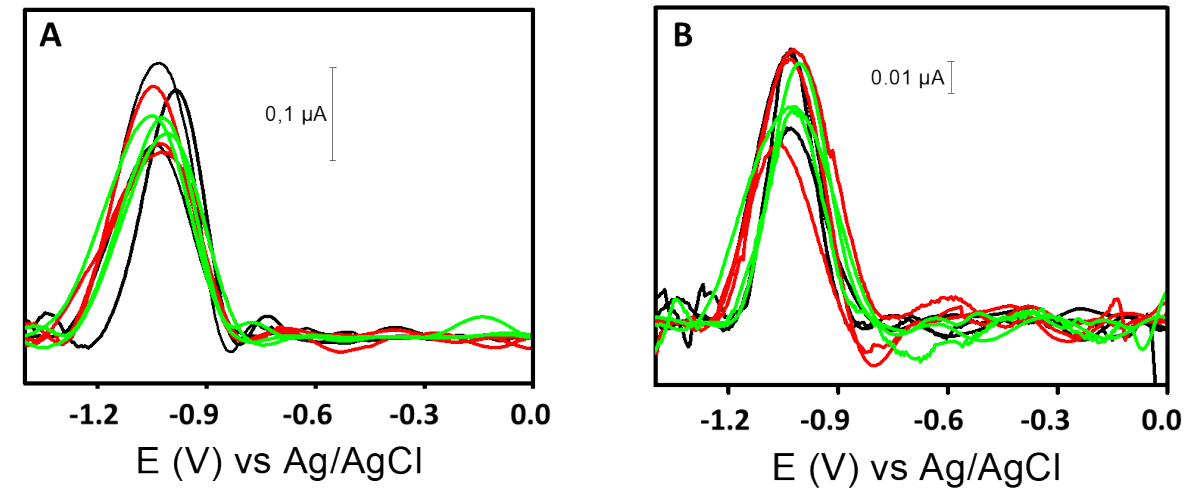

Figure S2. Voltammograms of A) $2000 \mathrm{mgL}^{-1} \mathrm{AGP}-\mathrm{Os}(\mathrm{VI})$ and B) $500 \mathrm{mgL}^{-1}$ of AGP$\mathrm{Os}(\mathrm{VI})$ by AdTSWV obtained from 3 days measurements (3 electrodes per day, $n=9$ ). Green line: 3 measurements day 1; red line: 3 measurements day 2; and black line: 3 measurements day 3 (electrochemical signals were baseline corrected and smoothed out). Conditions: as in Fig.S1 